\title{
Profile of nintedanib in the treatment of solid tumors: the evidence to date
}

This article was published in the following Dove Press journal:

OncoTargets and Therapy

8 December 2015

Number of times this article has been viewed

\author{
Niranjan Awasthi ${ }^{\prime}$ \\ Roderich E Schwarz ${ }^{1,2}$ \\ 'Department of Surgery, Indiana \\ University School of Medicine, South \\ Bend, IN, USA; ${ }^{2}$ Indiana University \\ Health Goshen Center for Cancer \\ Care, Goshen, IN, USA
}

\begin{abstract}
Angiogenesis is an essential process for tumor growth and metastasis, and remains a promising therapeutic target process in cancer treatment for several cancer types. Bevacizumab, a monoclonal antibody that targets vascular endothelial growth factor (VEGF), was the first antiangiogenic agent approved for cancer therapy. Novel antiangiogenic agents, such as sunitinib, sorafenib, pazopanib, or vandetanib that target additional proangiogenic signaling pathways beyond VEGF, have also been approved for the treatment of various malignant diseases. While most of these agents are approved in combination with cytotoxic chemotherapy for indications including metastatic colorectal cancer, non-small-cell lung cancer, breast cancer, renal cell carcinoma (RCC), and gastric cancer, some are used as approved monotherapy for advanced RCC, hepatocellular carcinoma and medullary thyroid cancer. Major challenges to the success of antiangiogenic therapy include associated toxicity risks, limitation of efficacy through the possible development of resistance and induction or promotion of metastatic progression. Nintedanib (formally known as BIBF 1120) is a triple angiokinase inhibitor of VEGF, fibroblast growth factor, platelet-derived growth factor signaling with lesser activity against RET, Flt-3, and Src. Through this unique targeting profile nintedanib has demonstrated significant antitumor activity in several tumor types in preclinical studies. Nintedanib has also shown promising clinical efficacy in combination with docetaxel and has been approved for treating patients with locally advanced and metastatic non-small-cell lung cancer in Europe. Nintedanib has also been found to be clinically promising in terms of efficacy and safety in several other solid tumors including ovarian cancer (Phase III), RCC (Phase II), and prostate cancer (Phase II). This review article provides a comprehensive summary of the preclinical and clinical efficacy of nintedanib in the treatment of solid tumors.
\end{abstract}

Keywords: nintedanib, BIBF 1120, angiogenesis, VEGF, tyrosine kinase inhibitors

\section{Introduction}

Angiogenesis, the process of new blood vessel development from existing vasculature, is a hallmark of cancer progression and metastasis that has long been considered an attractive therapeutic target. ${ }^{1}$ Tumor angiogenesis is a complex process that represents a highly regulated yet disturbed balance between proangiogenic and antiangiogenic mechanisms. ${ }^{2}$ Among several proangiogenic signaling molecules, vascular endothelial growth factor (VEGF) is considered to be one of the most important factors involved in tumor angiogenesis. ${ }^{3}$ VEGF is frequently highly expressed in human solid cancers and plays a fundamental role in tumor-mediated blood vessel growth by mediating vasculogenesis, angiogenic remodeling, angiogenic sprouting, and vascular permeability. ${ }^{3-5}$ Thus, initial antiangiogenic therapy attempts have largely focused on inhibiting VEGF signaling. Bevacizumab, a monoclonal antibody that blocks angiogenesis by binding VEGF-A (a ligand for VEGFR1 and VEGFR2), was the first antiangiogenic
Correspondence: Niranjan Awasthi Department of Surgery, Indiana University School of Medicine, $1234 \mathrm{~N}$ Notre Dame Avenue, South Bend, IN 466I7, USA

$\mathrm{Tel}+\mathrm{I} 57463 \mid 5780$

Fax +| $57463|782|$

Email nawasthi@iupui.edu 
agent approved in 2004 that showed clinical efficacy in combination with chemotherapy in some solid tumors including metastatic colorectal cancer (CRC), ${ }^{6}$ non-smallcell lung cancer (NSCLC), ${ }^{7}$ breast cancer, ${ }^{8}$ and renal cell carcinoma (RCC). ${ }^{9}$ Since then, several antiangiogenic agents, such as sunitinib, ${ }^{10}$ sorafenib,${ }^{11}$ pazopanib, ${ }^{12}$ vandetanib,${ }^{13}$ axitinib, ${ }^{14}$ regorafenib, ${ }^{15}$ aflibercept,${ }^{16}$ cabozantinib, ${ }^{17}$ and ramucirumab $^{18}$ that target VEGF signaling and/or some additional proangiogenic signaling activity have been approved for the treatment of various malignant diseases. These antiangiogenic agents, either as monotherapy or in combination with chemotherapy, generally have only provided limited clinical benefits in some tumor types. Furthermore, in certain advanced metastatic cancers, VEGF inhibition alone was insufficient to prevent progression, induced resistance and in some cases may have contributed to increased invasion and metastasis. ${ }^{19,20}$ One of the main reasons for the limited and transient response of the anti-VEGF therapies is that tumor angiogenesis is regulated by multiple pathways that are able to compensate for each other when single pathways are inhibited. The fibroblast growth factor (FGF) and its receptors (FGFR1/2/3), ${ }^{21}$ platelet-derived growth factor (PDGF), and its receptors (PDGFR $\alpha$ and PDGFR $\beta$ ), ${ }^{22}$ epidermal growth factor and its receptor, ${ }^{23}$ angiopoietin-2, ${ }^{24}$ placenta-derived growth factor, ${ }^{25}$ and neuropilin ${ }^{26}$ are key components within the main signaling pathways that provide potential escape mechanisms from anti-VEGF therapy leading to resistance development and facilitate resumption of tumor growth. ${ }^{27}$

The FGF/FGFR signaling axis plays an important role in tissue homeostasis, tissue repair, angiogenesis, and inflammation. Deregulation of FGF/FGFR signaling through genetic modification or over-expression of its ligands/receptors has been observed to promote cell proliferation, survival, and tumor angiogenesis in numerous tumor settings including pancreatic cancer, prostate cancer, and some squamous cell carcinomas. $^{28,29}$ Several preclinical studies demonstrated that inhibition of FGF/FGFR signaling has antiproliferative, proapoptotic, and antiangiogenic effects, supporting the validity of this signaling axis as a potential therapeutic target. PDGF is another important growth factor that can be crucial for tumor growth and progression. PDGF itself is a potent mitogen in both normal and tumor cells, ${ }^{30}$ but it also has significant angiogenic effects on endothelial cells. PDGF/PDGFR signaling has been shown to promote cell division, ${ }^{31,32}$ cell migration, ${ }^{33}$ and angiogenesis. ${ }^{34}$ In addition, recruitment of pericytes such as vascular smooth muscle cells by PDGF is essential for maintenance of tumor angiogenesis. ${ }^{35}$ The PDGF/PDGFR signaling has been overactive in several malignancies such as brain tumor, sarcomas, prostate cancer, liver cancer, NSCLC, breast cancer, and $\mathrm{CRC}^{36,37}$ and therapeutic targeting of this pathway reduced tumor growth in many tumor types. ${ }^{37-39}$

As multiple proangiogenic factors contribute to tumor angiogenesis, and the fact that inhibition of one angiogenic signaling pathway induces compensatory mechanisms leading to the development of resistance, ${ }^{40-42}$ more recent therapeutic strategies have focused on developing multi-targeted tyrosine kinase receptors (TKIs) that have the ability to block multiple signaling pathways simultaneously, but at the same time maintain some selectivity profile of kinases in terms of safety and tolerability. This review summarizes the antitumor profile of nintedanib, a triple angiokinase inhibitor, which targets proangiogenic signaling of VEGFR, FGFR, and PDGFR.

\section{Nintedanib: advantages and mechanism of action}

The first commercially available antiangiogenic drug was bevacizumab, a monoclonal antibody against VEGF-A. ${ }^{43}$ Bevacizumab is currently approved in combination with standard chemotherapy for the treatment of several cancers including metastatic CRC, ${ }^{6}$ NSCLC, ${ }^{7}$ and RCC. ${ }^{6,719}$ While bevacizumab demonstrates some promising antitumor activity, its clinical use may also be limited by its side-effect profile that includes hypertension, proteinuria, bleeding, thrombotic events, and hemorrhage. ${ }^{44,45}$ Efforts beyond bevacizumab have focused on a second class of antiangiogenic agents, namely small molecules that are orally available and have the ability to target multiple TKIs. Most of these broad-spectrum TKIs such as sunitinib, sorafenib, pazopanib, vandetanib have complex efficacy data, but in many cases the use of these drugs is associated with a significant increase in the incidence and risk of side effects. ${ }^{46-48}$ Most of these TKIs that affect multiple kinases have lower specificity toward some of their therapeutic targets, particularly at the FGF/ FGFR axis, requiring higher doses for efficacy. ${ }^{49}$ Therefore, these TKIs have increased risk for on-target toxicities (such as hypertension, skin toxicity, and diarrhea) and presumed off-target toxicities (such as cardiac impairment). ${ }^{49,50}$

Nintedanib is a next generation, oral, and potent triple angiokinase inhibitor that inhibits the proangiogenic pathways mediated by VEGFR1/2/3 ( $\left.\mathrm{IC}_{50} 13-34 \mathrm{nmol} / \mathrm{L}\right)$, FGFR1/2/3 $\left(\mathrm{IC}_{50} 37-108 \mathrm{nmol} / \mathrm{L}\right)$, and PDGFR $\alpha / \beta\left(\mathrm{IC}_{50} 59-65 \mathrm{nmol} / \mathrm{L}\right)$ to a high degree of specificity (Figure 1). Nintedanib competitively binds to the ATP binding-pocket of these receptors, resulting in interference with receptor dimerization and blocking intracellular signaling critical for the proliferation and survival of angiogenesis-related endothelial cells, pericytes, and vascular smooth muscle cells (Figure 2). Nintedanib also inhibits some 
A

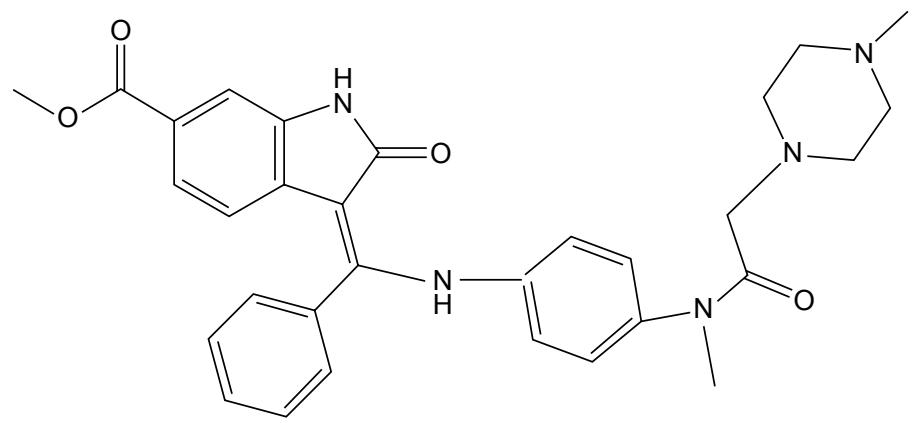

Nintedanib (BIBF 1120)

Chemical name: (Z)-methyl 3-((4-(N-methyl-2-(4-methylpiperazin-1-yl) acetamido)phenylamino)(phenyl)methylene)-2-oxoindoline-6-carboxylate

\begin{tabular}{|ll|}
\hline Kinase & IC $_{50}(\mathbf{n M})$ \\
VEGFR1 & $34 \pm 15$ \\
VEGFR2 & $21 \pm 13$ \\
VEGFR3 & $13 \pm 10$ \\
FGFR1 & $69 \pm 70$ \\
FGFR2 & $37 \pm 2$ \\
FGFR3 & $108 \pm 41$ \\
PDGFR $\alpha$ & $59 \pm 71$ \\
PDGFR $\beta$ & $65 \pm 7$ \\
IGF1R & $>1,000$ \\
InsR & $>4,000$ \\
FIt-3 & 26 \\
Lck & $16 \pm 16$ \\
Src & $156 \pm 40$ \\
Lyn & $195 \pm 12$ \\
EGFR, HER2, CDK1/2/4 & $>50,000$ \\
\hline
\end{tabular}

Figure I Summary of nintedanib.

Notes: (A) Molecular structure of nintedanib. (B) In vitro kinase inhibition profile of nintedanib $\left(\mathrm{IC}_{50}\right)$. Data presented as mean \pm standard error of at least three determinations.

Abbreviations: VEGFR, vascular endothelial growth factor receptor; FGFR, fibroblast growth factor receptor; PDGFR, platelet-derived growth factor receptor; IGFIR, insulin-like growth factor I receptor; InsR, insulin receptor; Flt-3, fms-like tyrosine protein kinase 3; Lck, lymphocyte-specific tyrosine kinase; Src, proto-oncogene tyrosine protein kinase src; Lyn, tyrosine-protein kinase lyn; EGFR, epidermal growth factor receptor; HER2, human epidermal growth factor receptor 2; CDK, cyclin-dependent kinase.

non-receptor kinases such as Fms-like tyrosine protein kinase (Flt-3, $\mathrm{IC}_{50} 26 \mathrm{nmol} / \mathrm{L}$ ), proto-oncogene ret (Ret, $\mathrm{IC}_{50} 35$ $\mathrm{nmol} / \mathrm{L}$ ), lymphocyte-specific tyrosine kinase (Lck, $\mathrm{IC}_{50} 16$ $\mathrm{nmol} / \mathrm{L}$ ), tyrosine-protein kinase lyn ( $\left.\mathrm{Lyn} \mathrm{IC}_{50} 195 \mathrm{nmol} / \mathrm{L}\right)$, and proto-oncogene tyrosine protein kinase src $\left(\mathrm{Src}, \mathrm{IC}_{50}\right.$ $156 \mathrm{nmol} / \mathrm{L})^{51,52}$ (Figure 1). In addition, nintedanib has recently been approved for the treatment of idiopathic pulmonary fibrosis based on results from the replicate Phase III trials involving 1,066 patients from 24 countries. ${ }^{53}$ Nintedanib is thus the first targeted treatment for idiopathic pulmonary fibrosis, indicating the therapeutic potential of TKIs in non-malignant diseases.

A major challenge in the success of VEGF signaling blocking antiangiogenic therapy is the development of resistance in the primary tumor, probably due to induction of tumor escape mechanisms through upregulated FGFR and PDGFR signaling. ${ }^{54-56}$ This mechanism of the development of antiangiogenic drug resistance provides a potential rationale for efficacy of nintedanib, which can prevent tumor growth and metastasis via its triple inhibition of VEGFR, FGFR and PDGFR, and also provide a therapeutic alternative for patients with intrinsic and acquired resistance to single or dual target antiangiogenic drugs.

\section{Nintedanib: preclinical studies to date}

In vitro studies demonstrated that nintedanib treatment inhibited the proliferation of VEGF-stimulated human umbilical vein endothelial cells and human skin microvascular endothelial cells. Nintedanib also inhibited the proliferation of PDGF-stimulated smooth muscle cells and pericytes. ${ }^{52}$
Although Kutluk Cenik et $\mathrm{al}^{57}$ showed that nintedanib has no antiproliferative activity on selected lung and pancreatic cancer cell lines, recent reports suggest that nintedanib effects are not generally limited to stromal cells as it also differentially inhibited the proliferation of hepatocellular carcinoma (HCC) cells ${ }^{58}$ and pancreatic ductal adenocarcinoma (PDAC) cells. ${ }^{59}$ Tai et $\mathrm{al}^{58}$ demonstrated that nintedanib treatment caused a significant antiproliferative effect in a panel of four HCC cell lines (PLC5, Hep3B, SK-Hep1, and HuH7) and one hepatoblastoma cell line (Hepg2). Awasthi et al ${ }^{59}$ demonstrated that nintedanib not only inhibited the proliferation of human umbilical vein endothelial cells, fibroblast WI-38 cells and pancreatic cancer stromal cells, but it also inhibited the proliferation of human PDAC cells (AsPC-1, BxPC-3, MIA $\mathrm{PaCa}-2$, and Panc-1) and murine PDAC cells PanO2.

In vivo, nintedanib demonstrated potent antitumor effects in all human tumor xenografts reported to date, including NSCLC, RCC, CRC, ovarian cancer, prostate cancer, and PDAC. ${ }^{52,57,59}$ More importantly, nintedanib treatment augmented the antitumor response of standard cytotoxic agents. In NSCLC xenografts (H460 cells), nintedanib treatment caused synergistic antitumor effects in combination with cytotoxic agents docetaxel or pemetrexed. ${ }^{60}$ Kutluk Cenik et al ${ }^{57}$ demonstrated significant antitumor activity of nintedanib monotherapy and in combination with standard cytotoxic chemotherapy in several xenograft models of lung and pancreatic cancer. ${ }^{57}$ Recently, Awasthi et al ${ }^{59}$ demonstrated that nintedanib significantly enhanced gemcitabine response in pancreatic cancer: net local tumor growth compared to 


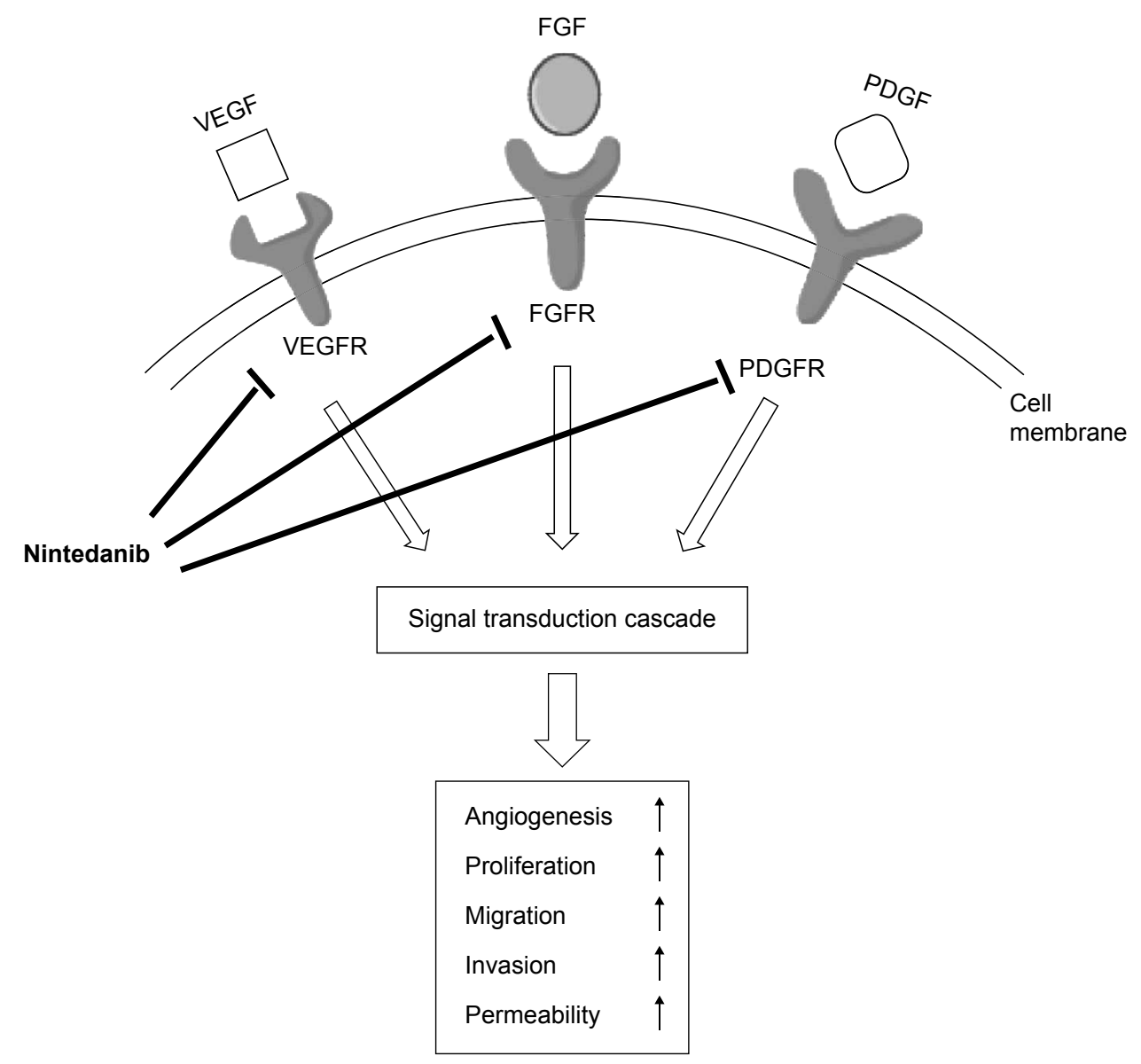

Figure 2 Triple angiokinase inhibition mechanism of nintedanib targeting tumor angiogenesis.

Abbreviations: VEGF, vascular endothelial growth factor; FGF, fibroblast growth factor; PDGF, platelet-derived growth factor.

controls $(100 \%)$ was $60.8 \% \pm 10.5 \%$ in the gemcitabine group, $-2.1 \% \pm 9.9 \%$ after nintedanib monotherapy and $-12.4 \% \pm 16 \%$ after gemcitabine plus nintedanib combination therapy. In addition, this study demonstrated that compared with controls, the increase in median animal survival was $56 \%$ with gemcitabine $(P=0.036), 94 \%$ for nintedanib $(P=0.004)$ and $138 \%$ after gemcitabine + nintedanib $(P=0.001) .{ }^{59}$ In human tumor xenografts, intratumoral antitumor mechanism of nintedanib involves reduced microvessel density, pericyte coverage, vessel permeability, tumor perfusion, and induced hypoxia, as well as inhibition of phosphatidylinositol 3-kinase (PI3K)-AKT and mitogen-activated protein kinase (MAPK) signaling pathways. . $2,57,59^{2}$

\section{Nintedanib: clinical studies to date}

The clinical pharmacokinetic profile studies of nintedanib demonstrated that it is rapidly absorbed as its maximum plasma concentrations occur within 1-3 hours after oral administration. ${ }^{61,62}$ The terminal half-life of nintedanib was observed to be $\sim 13-19$ hours. ${ }^{61,62}$ The first Phase I dose- escalation study with single agent nintedanib therapy was reported in 61 patients with different advanced solid tumors. ${ }^{62}$ Twenty-five patients received nintedanib $50-450 \mathrm{mg}$ once daily (od) and 36 patients received nintedanib $150-300 \mathrm{mg}$ twice daily (bid) in 4-week cycles with a week off. The most common drug-related adverse events (AEs) were mild to moderate; grade 3 or more AEs for nintedanib od vs bid occurring in $>5 \%$ patients were reversible hepatic enzyme elevation (12\% grade 3 and $4 \%$ grade 4 vs $0 \%$ grade 3 and $2.8 \%$ grade 4 ), increase in aspartate aminotransferase (AST; grade $3,8 \%$ vs $2.8 \%$ ), increase in alanine aminotransferase (ALT; grade 3, 0\% vs 5.6\%), increase in $\gamma$-glutamyl transpeptidase (grade $3,4 \%$ vs $5.6 \%$ ), decrease in CD-4 lymphocyte (grade $3,16 \%$ vs $5.6 \%$ ), hypertension (grade 3, $4 \%$ vs $0 \%$ ), diarrhea (grade $3,0 \%$ vs $2.8 \%$ ), nausea (grade $3,0 \%$ vs $5.6 \%$ ), and vomiting (grade $3,0 \%$ vs $2.8 \%$ ). The maximum tolerable dose (MTD) of nintedanib was defined as $250 \mathrm{mg}$ for both od and bid dosing. ${ }^{62}$ In another Phase I dose-escalation study, 21 Japanese patients with advanced refractory solid tumors were treated with bid nintedanib doses of $150 \mathrm{mg}$ 
$(\mathrm{n}=3), 200 \mathrm{mg}(\mathrm{n}=12)$, or $250 \mathrm{mg}(\mathrm{n}=6) .{ }^{63}$ This study reported that the dose-limiting grade 3 or 4 toxicity elevation of liver enzymes occurred in 3 of 12 patients at $200 \mathrm{mg}$ bid and 3 of 6 patients at $250 \mathrm{mg}$ bid dose. Stable disease (SD) was reported in $76.2 \%$ of patients $(n=16)$. The MTD of nintedanib in this study was determined to be $200 \mathrm{mg}$ bid.

\section{Nintedanib in NSCLC}

In NSCLC patients, two Phase I dose-escalation studies have been conducted investigating nintedanib in combination with a standard chemotherapy regimen. The first study investigated the MTD of continuous nintedanib oral treatment in combination with standard-dose pemetrexed $\left(500 \mathrm{mg} / \mathrm{m}^{2}\right)$ in patients who had been previously treated with one platinum-based chemotherapy regimen. ${ }^{64}$ In this study, one patient achieved a complete response after 44 days for more than 3 years; otherwise, the best overall response was $\mathrm{SD}$ in $50 \%$ patients $(\mathrm{n}=13)$. The second trial investigated the safety, tolerability, and MTD of nintedanib in combination with carboplatin and paclitaxel in patients with advanced NSCLC who were previously untreated. ${ }^{65}$ In these two Phase I studies, the recommended dose of nintedanib was determined as $200 \mathrm{mg}$ bid when used in combination with the mentioned standard chemotherapy regimens of NSCLC. The most frequent AEs in these studies were comparable to the nintedanib monotherapy trials.

A Phase II double-blind trial evaluated the efficacy of nintedanib monotherapy ( $150 \mathrm{mg}$ bid, $\mathrm{n}=36$ or $250 \mathrm{mg}$ bid, $\mathrm{n}=37$ ) in patients with relapsed, advanced NSCLC (any histology) with an Eastern Cooperative Oncology Group performance status $0-2$. This study reported a median progression free survival (PFS) of 6.9 weeks, the median overall survival (OS) was 21.9 weeks, and SD was observed in $46 \%$ of the patients (Table 1). In 56 patients with Eastern Cooperative Oncology Group performance status 0-1, median PFS was 11.6 weeks with a median OS of 37.7 weeks. Most commonly reported drug-related AEs included nausea, diarrhea, vomiting, anorexia, and abdominal pain. ${ }^{66}$

A Phase III, double-blind, randomized trial of nintedanib (200 mg, bid, $n=565)$ or placebo $(n=569)$ on days $2-21$, in combination with docetaxel $\left(75 \mathrm{mg} / \mathrm{m}^{2}\right.$ on day 1$)$ in patients with previously treated NSCLC (LUME-Lung 1 trial), reported that median PFS was significantly improved in the docetaxel plus nintedanib group compared with the docetaxel plus placebo group (3.4 months vs 2.7 months, hazard ratio (HR) $0.79, P=0.0019$ ). The median OS was also significantly improved in the docetaxel plus nintedanib group compared with docetaxel alone group (12.6 months vs 10.3 months;

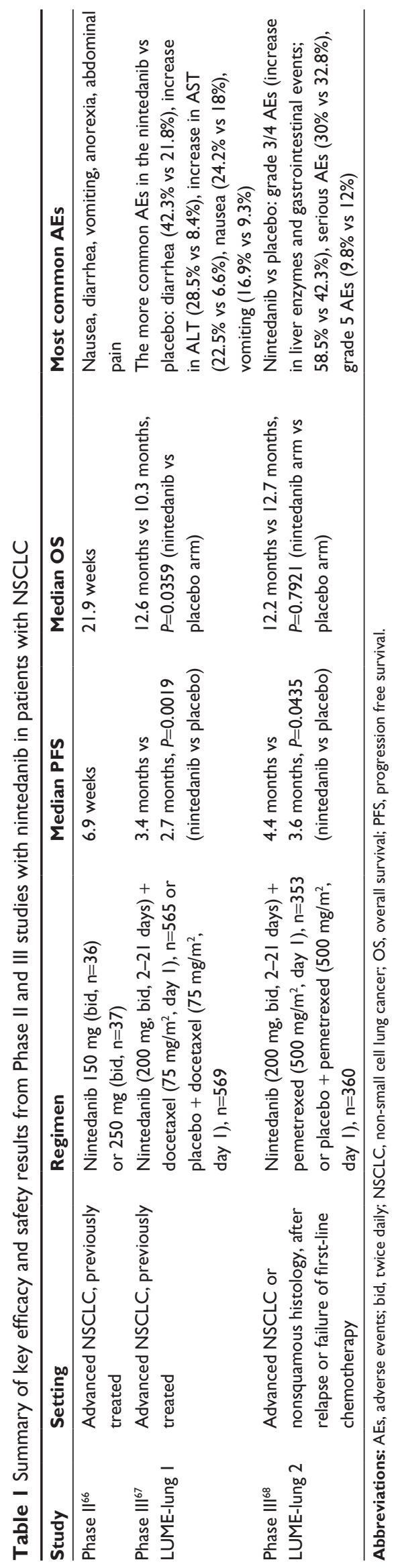




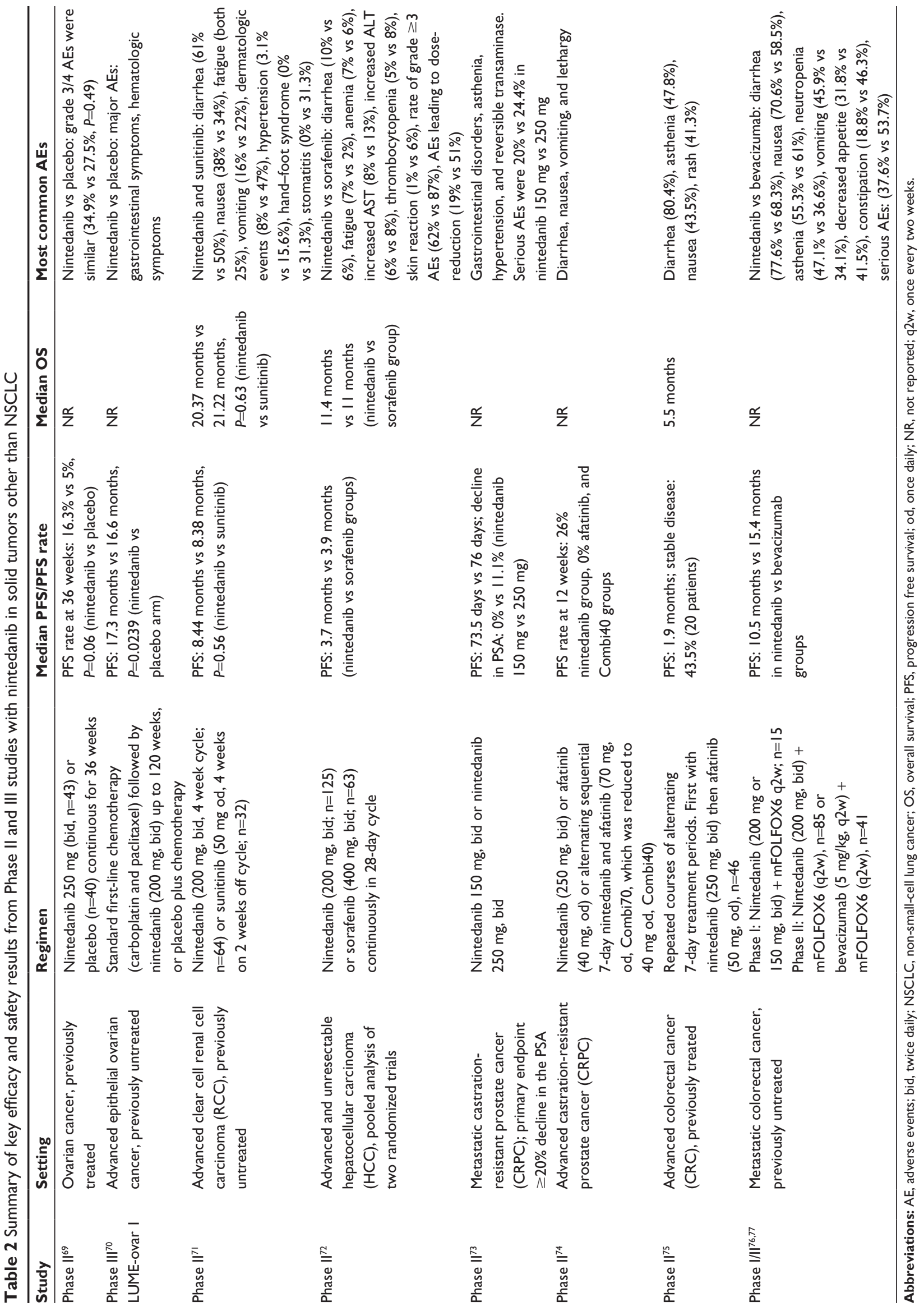


HR 0.83, $P=0.0359$ ) for all patients with adenocarcinoma histology. In the total study population, median OS in the docetaxel plus nintedanib group and docetaxel alone group was 10.1 and 9.1 months (HR 0.94, $P=0.2720$ ), respectively. Nintedanib plus docetaxel had a manageable safety profile as AEs more common in the combination group than in the docetaxel alone group included diarrhea (42.3\% vs $21.8 \%)$, increases in ALT (28.5\% vs 8.4\%), increase in AST (22.5\% vs $6.6 \%$ ), nausea $(24.2 \%$ vs $18 \%$ ), and vomiting ( $16.9 \%$ vs 9.3\%) (Table 1). Most of these AEs were manageable by symptom management and/or dose reduction. ${ }^{67}$ Based on the positive results of this study of 1,300 patients in 27 countries, the European Union granted approval for nintedanib in combination with docetaxel for use in patients with locally advanced, metastatic, or locally recurrent NSCLC with adenocarcinoma histology and progression after first-line chemotherapy. Another Phase III study (LUME-Lung 2) trial investigated the efficacy and safety of nintedanib (200 $\mathrm{mg}$, bid on 2-21 days) in combination with standard pemetrexed $\left(500 \mathrm{mg} / \mathrm{m}^{2}\right.$ on day 1) compared with pemetrexed alone $\left(500 \mathrm{mg} / \mathrm{m}^{2}\right.$ on day 1$)$ in patients with advanced or recurrent nonsquamous NSCLC after relapse or failure of first-line chemotherapy. In this study, patient enrollment was halted after 713 patients based on a pre-defined futility analysis of investigator-assessed PFS by an independent data monitoring committee. This decision was based on the likelihood that the primary endpoint of centrally assessed PFS would not be met, and was not safety related. Interestingly, a subsequent analysis of the 713 patients who were enrolled, demonstrated that the primary end-point of centrally reviewed PFS was met even though the study was stopped prematurely. This analysis showed that median PFS was significantly improved in the pemetrexed plus nintedanib group compared with pemetrexed alone (4.4 vs 3.6 months, HR $0.83, P=0.0435)$. There was no difference in OS between the two treatment groups (12.2 vs 12.7 months, HR 1.03, $P=0.7921)$ and overall response rates were also comparable $(9.1 \%$ vs $8.3 \%)$. There was a higher incidence of grade $3 / 4$ AEs (increase in liver enzymes and gastrointestinal events, overall $58.5 \%$ vs $42.3 \%$ ), serious AEs were $30 \%$ vs $32.8 \%$ and grade 5 AEs were $9.8 \%$ vs $12 \%$ in the nintedanib arm vs placebo (Table 1). ${ }^{68}$ A Phase II study (a biomarker-driven study: FGFR1 amplification as predictive of efficacy) of nintedanib in advanced squamous cell lung cancer patients who have failed up to two prior chemotherapy regimens is currently ongoing (NCT01948141). Another placebo-controlled, Phase III trial of nintedanib plus docetaxel in patients with stage IIIB/IV or recurrent adenocarcinoma NSCLC after failure of first line chemotherapy is also ongoing (LUMEColumbus, NCT02231164).

\section{Nintedanib in ovarian cancer}

In a Phase II double-blind, randomized trial of 83 patients who had just completed chemotherapy for relapsed ovarian cancer with evidence of response but at high risk of further early recurrence, patients were randomized to receive either nintedanib $250 \mathrm{mg}(\mathrm{n}=43)$ or placebo $(\mathrm{n}=40)$, bid, continuously for 36 weeks. The PFS rates at 36 weeks were $16.3 \%$ and $5 \%$ in the nintedanib and placebo groups, respectively (HR $0.65,95 \%$ CI: $0.42-1.02, P=0.06$ ). Grade 3 or 4 AEs were similar between the nintedanib group and placebo (34.9\% vs $27.5 \%, P=0.49$ ) (Table 2 ). This study concluded that nintedanib is well tolerated and it is associated with a potential improvement in survival. ${ }^{69}$

In a Phase III study (LUME-Ovar 1 or AGO-OVAR 12), nintedanib $200 \mathrm{mg}$ bid was added to standard first-line chemotherapy (carboplatin and paclitaxel) followed by nintedanib maintenance therapy for a maximum of 120 weeks as firstline treatment in patients with advanced epithelial ovarian cancer. This study demonstrated a significant improvement in median PFS in the nintedanib plus chemotherapy group compared with placebo/chemotherapy alone (17.3 vs 16.6 months, HR 0.84, 95\% CI: 0.72-0.98, $P=0.0239$ ) (Table 2). In a subgroup analysis, a more pronounced PFS benefit was observed with nintedanib compared with the placebo arm in patients with $<1 \mathrm{~cm}$ postsurgical residual tumor deposits (27.1 vs 20.8 months, HR 0.75, 95\% CI: 0.61-0.92, $P=0.005)$. This study is still ongoing..$^{70}$ Three Phase II trials of nintedanib are currently ongoing: one with and without metronomic dose of cyclophosphamide in advanced ovarian cancer (NCT01610869), one in combination with first line chemotherapy with interval debulking surgery in patients with ovarian cancer (NCT01583322), and another in bevacizumab resistant, persistent, or recurrent epithelial ovarian cancer (NCT01669798).

\section{Nintedanib in renal cell carcinoma}

The efficacy and safety of nintedanib (200 mg, bid, given in 4 week cycle, $n=64$ ) was assessed in a Phase II study of previously untreated advanced RCC patients and compared with the standard of care agent sunitinib (50 mg od, 4 weeks on, 2 weeks off schedule, $n=32$ ) as first-line systemic therapy. No significant difference in median PFS was observed between nintedanib and sunitinib treated groups (8.44 vs 8.38 months, HR $1.16,95 \%$ CI: $0.71-1.89, P=0.56$ ). Also, no significant difference was observed between the two therapy 
groups in terms of median OS (20.37 vs 21.22 months, HR $0.92,95 \%$ CI: $0.54-0.156, P=0.63)$ or 9 month PFS $(43 \%$ vs $45 \%, P=0.85$ ). The overall incidence of AE (any grade) was similar between nintedanib and sunitinib groups $(90.6 \%$ vs $93.8 \%$ ). However, grade 3 or more AEs occurred in $47 \%$ of patients after nintedanib compared with $56 \%$ after sunitinib. Most common AEs of all grades after nintedanib compared to sunitinib included diarrhea (61\% vs $50 \%$ ), nausea (38\% vs $34 \%$ ), fatigue (both $25 \%$ ), and vomiting (16\% vs $22 \%$ ). However, dermatologic AEs ( $8 \%$ vs 47\%) and other common AEs associated with antiangiogenic agents such as hypertension ( $3.1 \%$ vs $15.6 \%)$, hand-foot syndrome ( $0 \%$ vs $31.3 \%$ ), and stomatitis ( $0 \%$ vs $31.3 \%$ ), were less common in nintedanib treated patients compared with sunitinib therapy (Table 2). ${ }^{71}$

\section{Nintedanib in HCC}

Two Phase II studies evaluated the efficacy and safety of nintedanib (200 mg, bid) in comparison with sorafenib (400 mg, bid) continuously in 28-day cycle, in patients with unresectable, advanced HCC in Europe (NCT0100434003) and Asia (NCT00987935). Pooled analysis of these two trials (180 patients) in Caucasian and Asian patients showed similar efficacy between nintedanib and sorafenib therapy groups in terms of median time to progression (TTP; 3.7 vs 3.9 months, HR 1.31, 95\% CI: 0.89-1.91), median OS (11.4 vs 11 months, HR 0.91, 95\% CI: 0.65-1.29), and overall response rates ( $4 \%$ vs $5 \%$ ). The most frequent ( $>5 \%$ of patients in any group) grade $\geq 3$ AEs after nintedanib compared to sorafenib included diarrhea ( $10 \%$ vs $6 \%$ ), fatigue ( $7 \%$ vs $2 \%$ ), anemia ( $7 \%$ vs $6 \%$ ), increased AST ( $8 \%$ vs $13 \%$ ) and ALT (6\% vs $8 \%$ ), thrombocytopenia ( $5 \%$ vs $8 \%$ ), and skin reaction $(1 \%$ vs $6 \%$ ). However, the rates of grade $\geq 3$ AEs ( $62 \%$ vs $87 \%$ ) and AEs leading to dose reduction (19\% vs 51\%) were lower in the nintedanib group compared to sorafenib (Table 2). ${ }^{72}$

\section{Nintedanib in prostate cancer}

Two doses of nintedanib (150 and $250 \mathrm{mg}$, bid) were evaluated in a randomized Phase II study in metastatic castrationresistant prostate cancer patients after progression with docetaxel. ${ }^{73}$ The primary endpoint, prostate-specific antigen (PSA) response rate ( $\geq 20 \%$ decline in PSA from baseline), was $0 \%$ in the nintedanib $150 \mathrm{mg}$ group and $11.1 \%$ in the nintedanib $250 \mathrm{mg}$ group $(P=0.12)$. However, nintedanib at $250 \mathrm{mg}$ showed at least $50 \%$ PSA reduction in $5.6 \%$ patients and the rate of PSA increase in this group was significantly decelerated on treatment vs before treatment $(P=0.002)$. The median PFS was similar between the two groups (73.5 vs 76 days, $P=0.3)$. AEs included gastrointestinal disorders, asthenia, hypertension, and reversible transaminase elevation. The incidence of serious AEs for nintedanib $150 \mathrm{mg}$ vs $250 \mathrm{mg}$, were $20 \%$ vs $24.4 \%$ (Table 2 ). ${ }^{73}$

Another Phase II study evaluated nintedanib and afatinib, an ErbB family blocker, in advanced castration-resistant prostate cancer patients; dosing included nintedanib $(250 \mathrm{mg}$, bid, $n=27$ ), afatinib (40 mg od, $n=13$ ), or alternating sequential 7-day nintedanib (250 mg, bid) and afatinib (70 mg od; Combi70), which was later termed Combi40 $(n=10)$ because the afatinib dose was reduced to $40 \mathrm{mg}$ od due to AEs. The primary end-point of the study, the progression-free rate at 12 weeks was $26 \%$ for nintedanib and $0 \%$ for the afatinib and Combi40 groups (Table 2). However, the median TTP was 31 days (95\% CI: 29-84) for nintedanib monotherapy, 29 days (95\% CI: 29-54) for afatinib monotherapy, and 57 days (95\% CI: 29-78) for Combi40. The median PFS results were the same as for median TTP. Two patients had a $\geq 50 \%$ decline in PSA, one each in the nintedanib and the Combi40 groups. The most common drug-related AEs were diarrhea, nausea, vomiting, and lethargy; the majority of patients $(>90 \%)$ in each group had at least one therapy-related AE. Rash was more frequent with afatinib and elevation in transaminase was more frequent with nintedanib monotherapy. The frequency of therapy-related AEs did not increase in Combi40 compared with monotherapy. ${ }^{74}$

\section{Nintedanib in CRC}

In a Phase II study an alternating regimen of nintedanib (250 mg, bid) and then afatinib (50 mg, od) was evaluated in patients with advanced pretreated CRC. In this study, the best response was SD in 20 patients (43.5\%) but no objective responses were observed. Seven patients $(15.2 \%)$ remained progression-free for $\geq 16$ weeks. Median PFS was 1.9 months; median OS was 5.5 months. The most common AEs were diarrhea (80.4\%), asthenia (47.8\%), nausea (43.5\%), and rash (41.3\%) (Table 2). ${ }^{75}$ In another Phase II study, nintedanib plus mFOLFOX6 compared to bevacizumab plus mFOLFOX6 were evaluated in 126 patients with previously untreated metastatic CRC. The primary endpoint of this study was the kaplan-meier (KM) estimate of PFS at 9 months, which was $62.1 \%$ for the nintedanib regimen and $70.2 \%$ for the bevacizumab regimen; a numerical difference in PFS between treatments was $-8.1 \%$ (95\% CI: -27.8 to 11.5$)$. The results for best confirmed overall response were 63.5\% (95\% CI: 52.4-73.7) for the nintedanib and 56.1\% (95\% CI: 39.7-71.5) for the bevacizumab group. SD frequency was lower in the nintedanib than in the bevacizumab group $(27.1 \%$ vs $36.6 \%$ ). The final analysis of this study demonstrated that the 
median PFS was 10.5 months in the nintedanib group (95\% CI: 9.4-12.4) and 15.4 months in the bevacizumab group (95\% CI: 9.6-18.9), which contrasted with a median PFS at the time of the interim analysis (nintedanib: 10.6 months, bevacizumab: 9.2 months). The most frequent AEs ( $>40 \%$ in either group) in the nintedanib vs bevacizumab groups were diarrhea (77.6\% vs $68.3 \%$ ), nausea ( $70.6 \%$ vs $58.5 \%$ ), asthenia (55.3\% vs $61 \%$ ), neutropenia ( $47.1 \%$ vs $36.6 \%)$, vomiting (45.9\% vs $34.1 \%)$, decreased appetite (31.8\% vs $41.5 \%$ ), and constipation ( $18.8 \%$ vs $46.3 \%)$. Incidences of AEs leading to discontinuation of nintedanib or bevacizumab, with or without discontinuation of mFOLFOX, were $27.1 \%$ for nintedanib and $31.7 \%$ in the bevacizumab group. The overall incidence of serious AEs was lower for the nintedanib group than for the bevacizumab group (37.6\% vs 53.7\%) (Table 2). ${ }^{76,77}$ A double-blind, randomized, Phase III study of nintedanib vs placebo in refractory CRC is currently ongoing (NCT02149108). Another Phase I/II study of nintedanib in combination with capecitabine in treating patients with refractory metastatic CRC is currently ongoing (NCT02393755).

\section{Nintedanib in breast cancer}

In a Phase I study, the combination of nintedanib (150 mg, bid) with a standard preoperative treatment for breast cancer (weekly $80 \mathrm{mg} / \mathrm{m}^{2}$ paclitaxel, followed by adriamycin plus cyclophosphamide) was evaluated in HER-2-negative breast cancer patients. ${ }^{78}$ At this dose level, the observed toxicity was not different from that reported with paclitaxel alone therapy. The dose-limiting toxicity was transaminase elevation. The dose intensity of both initial drugs, nintedanib and paclitaxel, was $99.9 \%$ and $97.4 \%$, respectively. Despite the low number of patients $(n=8)$, the efficacy at this dose level was very promising with a pathological complete response of $50 \%$. Based on these trial data, a randomized Phase II study of docetaxel with or without nintedanib in patients receiving a second-line of chemotherapy for neoadjuvant HER-2negative breast cancer patients is ongoing (NCT01658462). Another Phase II study of nintedanib monotherapy for patients with metastatic HER2-negative inflammatory breast cancer is also ongoing (NCT02389764).

Based on promising efficacy and safety results of nintedanib in the above-mentioned solid tumors, nintedanib is currently under investigation in several other solid tumors including thyroid cancer (NCT01788982), neuroendocrine tumors (NCT02399215), cervix cancer (NCT02009579), endometrial cancer (NCT01225887), esophagogastric cancer (NCT02234596), urothelial carcinoma (NCT02278978), and glioblastoma (NCT01666600).

\section{Conclusion}

Increased understanding of the complexity of tumor angiogenesis pathways indicated the importance of multitargeted antiangiogenic agents to increase tumor response and overcome or delay resistance development. By simultaneous targeting of VEGF, FGF, and PDGF signaling, nintedanib may offer a better approach for the treatment of several solid tumors. The clinical efficacy of nintedanib in combination with docetaxel in NSCLC was recognized by its approval in the European Union. Nintedanib also demonstrated rather encouraging clinical efficacy and tolerability data in patients with different solid tumors, and several additional clinical studies are currently ongoing. There is a good possibility that nintedanib will be approved for at least some of these tumor types in the near future. However, more understanding of nintedanib's molecular mechanism of action and identifying molecular biomarkers to predict nintedanib response is highly desired to maximize clinical benefits.

\section{Disclosure}

The authors report no conflicts of interest in this work.

\section{References}

1. Folkman J. Tumor angiogenesis: therapeutic implications. $N$ Engl $J$ Med. 1971;285(21):1182-1186.

2. Al-Husein B, Abdalla M, Trepte M, Deremer DL, Somanath PR. Antiangiogenic therapy for cancer: an update. Pharmacotherapy. 2012; 32(12):1095-1111.

3. Moreira IS, Fernandes PA, Ramos MJ. Vascular endothelial growth factor (VEGF) inhibition - a critical review. Anticancer Agents Med Chem. 2007;7(2):223-245.

4. Ferrara N. Vascular endothelial growth factor as a target for anticancer therapy. Oncologist. 2004;9(Suppl 1):2-10.

5. Shojaei F, Ferrara N. Antiangiogenic therapy for cancer: an update. Cancer J. 2007;13(6):345-348.

6. Giantonio BJ, Catalano PJ, Meropol NJ, et al. Bevacizumab in combination with oxaliplatin, fluorouracil, and leucovorin (FOLFOX4) for previously treated metastatic colorectal cancer: results from the Eastern Cooperative Oncology Group Study E3200. J Clin Oncol. 2007;25(12): 1539-1544.

7. Sandler A, Gray R, Perry MC, et al. Paclitaxel-carboplatin alone or with bevacizumab for non-small-cell lung cancer. N Engl J Med. 2006; 355(24):2542-2550

8. Miller K, Wang M, Gralow J, et al. Paclitaxel plus bevacizumab versus paclitaxel alone for metastatic breast cancer. $N$ Engl J Med. 2007; 357(26):2666-2676.

9. Rini BI, Halabi S, Rosenberg JE, et al. Phase III trial of bevacizumab plus interferon alfa versus interferon alfa monotherapy in patients with metastatic renal cell carcinoma: final results of CALGB 90206. J Clin Oncol. 2010;28(13):2137-2143.

10. Motzer RJ, Hutson TE, Tomczak P, et al. Sunitinib versus interferon alfa in metastatic renal-cell carcinoma. $N$ Engl J Med. 2007;356(2): $115-124$.

11. Escudier B, Eisen T, Stadler WM, et al. Sorafenib in advanced clear-cell renal-cell carcinoma. $N$ Engl J Med. 2007;356(2):125-134.

12. Sternberg CN, Davis ID, Mardiak J, et al. Pazopanib in locally advanced or metastatic renal cell carcinoma: results of a randomized phase III trial. J Clin Oncol. 2010;28(6):1061-1068. 
13. Wells SA Jr, Robinson BG, Gagel RF, et al. Vandetanib in patients with locally advanced or metastatic medullary thyroid cancer: a randomized, double-blind phase III trial. J Clin Oncol. 2012;30(2):134-141.

14. Motzer RJ, Escudier B, Tomczak P, et al. Axitinib versus sorafenib as second-line treatment for advanced renal cell carcinoma: overall survival analysis and updated results from a randomised phase 3 trial. Lancet Oncol. 2013;14(6):552-562.

15. Grothey A, Van Cutsem E, Sobrero A, et al. Regorafenib monotherapy for previously treated metastatic colorectal cancer (CORRECT): an international, multicentre, randomised, placebo-controlled, phase 3 trial. Lancet. 2013;381(9863):303-312.

16. Van Cutsem E, Tabernero J, Lakomy R, et al. Addition of aflibercept to fluorouracil, leucovorin, and irinotecan improves survival in a phase III randomized trial in patients with metastatic colorectal cancer previously treated with an oxaliplatin-based regimen. J Clin Oncol. 2012; 30(28):3499-3506.

17. Elisei R, Schlumberger MJ, Muller SP, et al. Cabozantinib in progressive medullary thyroid cancer. J Clin Oncol. 2013;31(29): 3639-3646.

18. Fuchs CS, Tomasek J, Yong CJ, et al. Ramucirumab monotherapy for previously treated advanced gastric or gastro-oesophageal junction adenocarcinoma (REGARD): an international, randomised, multicentre, placebo-controlled, phase 3 trial. Lancet. 2014;383(9911):31-39.

19. Rini BI, Michaelson MD, Rosenberg JE, et al. Antitumor activity and biomarker analysis of sunitinib in patients with bevacizumabrefractory metastatic renal cell carcinoma. J Clin Oncol. 2008;26(22): 3743-3748.

20. Ebos JM, Lee CR, Cruz-Munoz W, Bjarnason GA, Christensen JG, Kerbel RS. Accelerated metastasis after short-term treatment with a potent inhibitor of tumor angiogenesis. Cancer Cell. 2009;15(3): 232-239.

21. Rusnati M, Presta M. Fibroblast growth factors/fibroblast growth factor receptors as targets for the development of anti-angiogenesis strategies. Curr Pharm Des. 2007;13(20):2025-2044.

22. Wu E, Palmer N, Tian Z, et al. Comprehensive dissection of PDGFPDGFR signaling pathways in PDGFR genetically defined cells. PLoS One. 2008;3(11):e3794.

23. Cascone T, Herynk MH, Xu L, et al. Upregulated stromal EGFR and vascular remodeling in mouse xenograft models of angiogenesis inhibitor-resistant human lung adenocarcinoma. J Clin Invest. 2011; 121(4): 1313-1328.

24. Rigamonti N, Kadioglu E, Keklikoglou I, Wyser Rmili C, Leow CC, De Palma M. Role of angiopoietin-2 in adaptive tumor resistance to VEGF signaling blockade. Cell Rep. 2014;8(3):696-706.

25. Fischer C, Jonckx B, Mazzone M, et al. Anti-P1GF inhibits growth of VEGF(R)-inhibitor-resistant tumors without affecting healthy vessels. Cell. 2007;131(3):46-475.

26. Ellis LM, Hicklin DJ. Pathways mediating resistance to vascular endothelial growth factor-targeted therapy. Clin Cancer Res. 2008;14(20): 6371-6375.

27. Abdullah SE, Perez-Soler R. Mechanisms of resistance to vascular endothelial growth factor blockade. Cancer. 2012;118(14): 3455-3467.

28. Beenken A, Mohammadi M. The FGF family: biology, pathophysiology and therapy. Nat Rev Drug Discov. 2009;8(3):235-253.

29. Presta M, Dell'Era P, Mitola S, Moroni E, Ronca R, Rusnati M. Fibroblast growth factor/fibroblast growth factor receptor system in angiogenesis. Cytokine Growth Factor Rev. 2005;16(2):159-178.

30. Heldin CH, Westermark B. Mechanism of action and in vivo role of platelet-derived growth factor. Physiol Rev. 1999;79(4):1283-1316.

31. Xie J, Aszterbaum M, Zhang X, et al. A role of PDGFRalpha in basal cell carcinoma proliferation. Proc Natl Acad Sci U S A. 2001;98(16): 9255-9259.

32. Funa K, Papanicolaou V, Juhlin C, et al. Expression of platelet-derived growth factor beta-receptors on stromal tissue cells in human carcinoid tumors. Cancer Res. 1990;50(3):748-753.
33. Bornfeldt KE, Raines EW, Nakano T, Graves LM, Krebs EG, Ross R. Insulin-like growth factor-I and platelet-derived growth factor-BB induce directed migration of human arterial smooth muscle cells via signaling pathways that are distinct from those of proliferation. J Clin Invest. 1994;93(3):1266-1274.

34. Kerbel RS. Tumor angiogenesis. N Engl J Med. 2008;358(19): 2039-2049.

35. Hellstrom M, Kalen M, Lindahl P, Abramsson A, Betsholtz C. Role of PDGF-B and PDGFR-beta in recruitment of vascular smooth muscle cells and pericytes during embryonic blood vessel formation in the mouse. Development. 1999;126(14):3047-3055.

36. Pietras K, Sjoblom T, Rubin K, Heldin CH, Ostman A. PDGF receptors as cancer drug targets. Cancer Cell. 2003;3(5):439-443.

37. Heldin $\mathrm{CH}$. Targeting the PDGF signaling pathway in tumor treatment. Cell Commun Signal. 2013;11:97.

38. Taeger J, Moser C, Hellerbrand C, et al. Targeting FGFR/PDGFR/ VEGFR impairs tumor growth, angiogenesis, and metastasis by effects on tumor cells, endothelial cells, and pericytes in pancreatic cancer. $\mathrm{Mol}$ Cancer Ther. 2011;10(11):2157-2167.

39. Kinoshita K, Nakagawa K, Hamada J, et al. Imatinib mesylate inhibits the proliferation-stimulating effect of human lung cancer-associated stromal fibroblasts on lung cancer cells. Int J Oncol. 2010;37(4): 869-877.

40. Erber R, Thurnher A, Katsen AD, et al. Combined inhibition of VEGF and PDGF signaling enforces tumor vessel regression by interfering with pericyte-mediated endothelial cell survival mechanisms. FASEBJ. 2004;18(2):338-340.

41. Bergers G, Song S, Meyer-Morse N, Bergsland E, Hanahan D. Benefits of targeting both pericytes and endothelial cells in the tumor vasculature with kinase inhibitors. J Clin Invest. 2003;111(9):1287-1295.

42. Casanovas O, Hicklin DJ, Bergers G, Hanahan D. Drug resistance by evasion of antiangiogenic targeting of VEGF signaling in late-stage pancreatic islet tumors. Cancer Cell. 2005;8(4):299-309.

43. Ferrara N, Hillan KJ, Gerber HP, Novotny W. Discovery and development of bevacizumab, an anti-VEGF antibody for treating cancer. Nat Rev Drug Discov. 2004;3(5):391-400.

44. Afranie-Sakyi JA, Klement GL. The toxicity of anti-VEGF agents when coupled with standard chemotherapeutics. Cancer Lett. 2015; 357(1):1-7.

45. Herbst RS. Toxicities of antiangiogenic therapy in non-small-cell lung cancer. Clin Lung Cancer. 2006;8(Suppl 1):S23-S30.

46. Iacovelli R, Palazzo A, Procopio G, et al. Incidence and relative risk of hepatic toxicity in patients treated with anti-angiogenic tyrosine kinase inhibitors for malignancy. Br J Clin Pharmacol. 2014;77(6): 929-938.

47. Cabebe E, Fisher GA. Clinical trials of VEGF receptor tyrosine kinase inhibitors in pancreatic cancer. Expert Opin Investig Drugs. 2007; 16(4):467-476

48. Arora A, Scholar EM. Role of tyrosine kinase inhibitors in cancer therapy. J Pharmacol Exp Ther. 2005;315(3):971-979.

49. Capdevila J, Carrato A, Tabernero J, Grande E. What could Nintedanib (BIBF 1120), a triple inhibitor of VEGFR, PDGFR, and FGFR, add to the current treatment options for patients with metastatic colorectal cancer? Crit Rev Oncol Hematol. 2014;92:83-106.

50. Broekman F, Giovannetti E, Peters GJ. Tyrosine kinase inhibitors: multitargeted or single-targeted? World J Clin Oncol. 2011;2(2):80-93.

51. Roth GJ, Heckel A, Colbatzky F, et al. Design, synthesis, and evaluation of indolinones as triple angiokinase inhibitors and the discovery of a highly specific 6-methoxycarbonyl-substituted indolinone (BIBF 1120). J Med Chem. 2009;52(14):4466-4480.

52. Hilberg F, Roth GJ, Krssak M, et al. BIBF 1120: triple angiokinase inhibitor with sustained receptor blockade and good antitumor efficacy. Cancer Res. 2008;68(12):4774-4782.

53. Richeldi L, du Bois RM, Raghu G, et al. Efficacy and safety of nintedanib in idiopathic pulmonary fibrosis. $N$ Engl J Med. 2014;370(22): 2071-2082. 
54. Ebos JM, Kerbel RS. Antiangiogenic therapy: impact on invasion, disease progression, and metastasis. Nat Rev Clin Oncol. 2011;8(4): 210-221.

55. Paez-Ribes M, Allen E, Hudock J, et al. Antiangiogenic therapy elicits malignant progression of tumors to increased local invasion and distant metastasis. Cancer Cell. 2009;15(3):220-231.

56. Sennino B, Ishiguro-Oonuma T, Wei Y, et al. Suppression of tumor invasion and metastasis by concurrent inhibition of c-Met and VEGF signaling in pancreatic neuroendocrine tumors. Cancer Discov. 2012; 2(3):270-287.

57. Kutluk Cenik B, Ostapoff KT, Gerber DE, Brekken RA. BIBF 1120 (nintedanib), a triple angiokinase inhibitor, induces hypoxia but not EMT and blocks progression of preclinical models of lung and pancreatic cancer. Mol Cancer Ther. 2013;12(6):992-1001.

58. Tai WT, Shiau CW, Li YS, et al. Nintedanib (BIBF-1120) inhibits hepatocellular carcinoma growth independent of angiokinase activity. J Hepatol. 2014;61(1):89-97.

59. Awasthi N, Hinz S, Brekken RA, Schwarz MA, Schwarz RE. Nintedanib, a triple angiokinase inhibitor, enhances cytotoxic therapy response in pancreatic cancer. Cancer Lett. 2015;358(1): 59-66.

60. Hilberg F, Brandstetter I. Efficacy of BIBF 1120, a potent triple angiokinase inhibitor, in models of human non-small cell lung cancer is augmented by chemotherapy. Eur J Cancer. 2007;2:S380.

61. Stopfer P, Rathgen K, Bischoff D, et al. Pharmacokinetics and metabolism of BIBF 1120 after oral dosing to healthy male volunteers. Xenobiotica. 2011;41(4):297-311.

62. Mross K, Stefanic M, Gmehling D, et al. Phase I study of the angiogenesis inhibitor BIBF 1120 in patients with advanced solid tumors Clin Cancer Res. 2010;16(1):311-319.

63. Okamoto I, Kaneda H, Satoh T, et al. Phase I safety, pharmacokinetic, and biomarker study of BIBF 1120, an oral triple tyrosine kinase inhibitor in patients with advanced solid tumors. Mol Cancer Ther. 2010; 9(10):2825-2833.

64. Ellis PM, Kaiser R, Zhao Y, Stopfer P, Gyorffy S, Hanna N. Phase I open-label study of continuous treatment with BIBF 1120, a triple angiokinase inhibitor, and pemetrexed in pretreated non-small cell lung cancer patients. Clin Cancer Res. 2010;16(10):2881-2889.

65. Doebele RC, Conkling P, Traynor AM, et al. A phase I, open-label dose-escalation study of continuous treatment with BIBF 1120 in combination with paclitaxel and carboplatin as first-line treatment in patients with advanced non-small-cell lung cancer. Ann Oncol. 2012;23(8): 2094-2102.

66. Reck M, Kaiser R, Eschbach C, et al. A phase II double-blind study to investigate efficacy and safety of two doses of the triple angiokinase inhibitor BIBF 1120 in patients with relapsed advanced non-small-cell lung cancer. Ann Oncol. 2011;22(6):1374-1381.
67. Reck M, Kaiser R, Mellemgaard A, et al. Docetaxel plus nintedanib versus docetaxel plus placebo in patients with previously treated non-small-cell lung cancer (LUME-Lung 1): a phase 3, double-blind, randomised controlled trial. Lancet Oncol. 2014;15(2):143-155.

68. Hanna NK, Kaiser R, Sullivan, RN, et al. Lume-lung 2: a multicenter, randomized, double-blind, phase III study of nintedanib plus pem- etrexed versus placebo plus pemetrexed in patients with advanced nonsquamous non-small cell lung cancer (NSCLC) after failure of firstline chemotherapy. J Clin Oncol. 2013;31(Suppl:8034).

69. Ledermann JA, Hackshaw A, Kaye S, et al. Randomized phase II placebo-controlled trial of maintenance therapy using the oral triple angiokinase inhibitor BIBF 1120 after chemotherapy for relapsed ovarian cancer. J Clin Oncol. 2011;29(28):3798-3804.

70. du Bois A, Kristensen G, Ray-Coquard I, et al. AGO-Ovar 12: a randomized placebo-controlled GCIG/ENGOT-intergroup phase III trial of standard frontline chemotherapy \pm nintedanib for advanced ovarian cancer. Int J Gynecol Cancer. 2013;23:PL01.

71. Eisen T, Shparyk Y, Jones R, et al. Phase II efficacy and safety study of nintedanib versus sunitinib in previously untreated renal cell carcinoma (RCC) patients. J Clin Oncol. 2013;31(abstract 4506).

72. Meyer T, Palmer DH, Chao Y, et al. Efficacy and safety study of nintedanib $(\mathrm{N})$ versus sorafenib $(\mathrm{S})$ in Caucasian and Asian patients with advanced hepatocellular carcinoma (HCC): pooled analysis of two randomized phase II trials. J Clin Oncol. 2015;33(abstract 4074).

73. Droz JP, Medioni J, Chevreau C, et al. Randomized phase II study of nintedanib in metastatic castration-resistant prostate cancer postdocetaxel. Anticancer Drugs. 2014;25(9):1081-1088.

74. Molife LR, Omlin A, Jones RJ, et al. Randomized Phase II trial of nintedanib, afatinib and sequential combination in castration-resistant prostate cancer. Future Oncol. 2014;10(2):219-231.

75. Bouche O, Maindrault-Goebel F, Ducreux M, et al. Phase II trial of weekly alternating sequential BIBF 1120 and afatinib for advanced colorectal cancer. Anticancer Res. 2011;31(6):2271-2281.

76. Van Cutsem E, Prenen H, Guillen-Ponce C, et al. A phase I/II, openlabel, randomised study of BIBF $1120 *$ plus mFOLFOX6 compared to bevacizumab plus mFOLFOX6 in patients with metastatic colorectal cancer. Eur J Cancer. 2011;47:8-9.

77. Boehringer Ingelheim BTNFR. A phase I-II study of BIBF 1120 and Folfox compared to Bevacizumab and Folfox in first line metastatic colorectal cancer patients. NLM identifier: 2008-005364-14. Available from: http://rials.boehringer-ingelheim.com/content/dam/internet/opu/ clinicaltrial/com_EN/results/1199/1199.51_U12-2578-02_DR.pdf. Accessed June 3, 2013.

78. Quintela-Fandino M, Urruticoechea A, Guerra J, et al. Phase I clinical trial of nintedanib plus paclitaxel in early HER-2-negative breast cancer (CNIO-BR-01-2010/GEICAM-2010-10 study). Br J Cancer. 2014; 111(6):1060-1064.
OncoTargets and Therapy

\section{Publish your work in this journal}

OncoTargets and Therapy is an international, peer-reviewed, open access journal focusing on the pathological basis of all cancers, potential targets for therapy and treatment protocols employed to improve the management of cancer patients. The journal also focuses on the impact of management programs and new therapeutic agents and protocols on

\section{Dovepress}

patient perspectives such as quality of life, adherence and satisfaction. The manuscript management system is completely online and includes a very quick and fair peer-review system, which is all easy to use. Visit http://www.dovepress.com/testimonials.php to read real quotes from published authors. 